\title{
Extracting Velocities for Faint Secondaries
}

\section{COHEN}

Department of Astronomy, University of Wisconsin, 475 Charter Street, Madison, Wisconsin 53706 USA

and

Harvard-Smithsonian Center for Astrophysics, 60 Garden Street, Cambridge, MA 02138, USA

D. W. LATHAM

Harvard-Smithsonian Center for Astrophysics, 60 Garden Street, Cambridge, MA 02138, USA

\begin{abstract}
When velocities can be determined for both members of a doublelined spectroscopic binary, then the mass ratio can be calculated immediately. With the goal of messuring velocities for faint secondaries in single-lined spectroscopic binaries, we have experimented with a technique of first subtracting the spectrum of the primary using synthetic spectra calculated from Kurucz model stellar atmospheres and then correlating to get the velocity of the secondary. For half a dozen of the single-lined spectroscopic binaries in the Carney-Latham survey of proper-motion stars we have been able to extract velocities for the secondaries and to solve for double-lined orbits.
\end{abstract}

Orbital solutions for double-lined spectroscopic binaries (DLSBs) are particularly useful when studying the distribution of secondary masses (or mass ratios) for a sample of spectroscopic binaries, because the mass ratio can be determined directly from the ratio of the observed velocity amplitudes. With orbital solutions for single-lined spectroscopic binaries (SLSBs), the distribution of secondary masses (or mass ratios) can only be analyzed statistically (e.g., Mazeh \& Goldberg 1992a, 1992b).

About $10 \%$ of the spectroscopic binaries discovered in the CfA radialvelocity surveys (Latham 1992) are obvious DLSBs and show two correlation peaks at phases when the velocities of the primary and secondary stars are well separated. When the secondary is more than 3 or 4 times fainter than the primary, the standard observing procedures and data reductions do not normally allow the secondary peak to be detected easily. For DLSBs composed of two dwarf stars, this means that an easily detected secondary must have nearly the same mass as its primary, because luminosity depends so strongly on mass. With the two goals of increasing the number of DLSBs discovered in the CfA surveys, and of extending the range of derived mass ratios, we have experimented with a technique for extracting velocities of faint secondaries. First we subtract the spectrum of the primary, and then we correlate the residual spectrum of the secondary against a template. For both steps we use synthetic spectra (Carney et al. 1987) calculated from Kurucz model atmospheres.

For almost 1500 stars in the Carney-Latham proper-motion survey, nearly 20,000 precise radial velocities have been accumulated over a period of about ten years. When we undertook the work reported here, orbital solutions were available for 108 spectroscopic binaries in this sample, 14 of which were DLSBs (Latham et al. 1992a, 1992b). For each SLSB in the sample we calculated the 
a

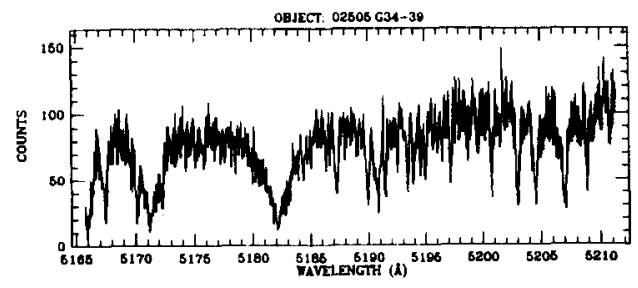

$b$

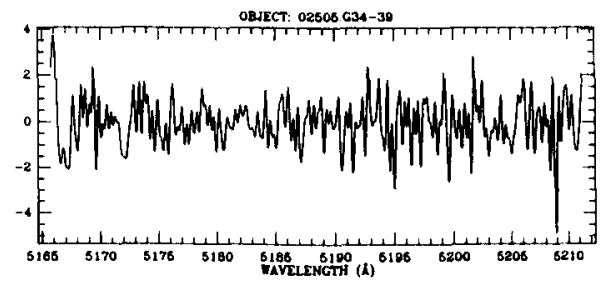

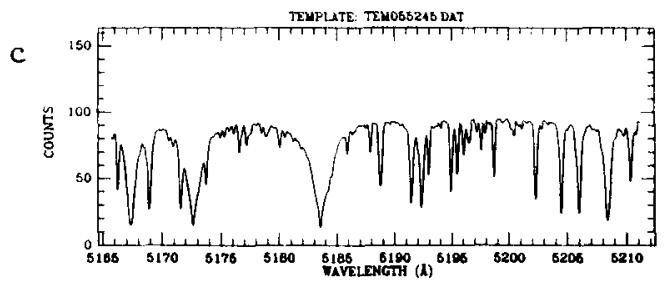

e
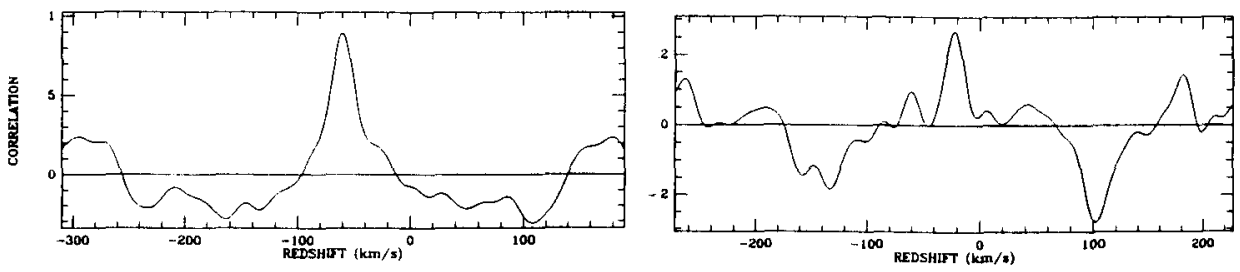

FIGURE 1. a. Observed spectrum. b. Calculated spectrum. c. Correlation of observed and calculated spectra. d. Residual secondary spectrum after subtraction of primary spectrum. e. Correlation of residual secondary spectrum and calculated spectra.

most probable mass ratio, following the precepts described by Torres (1991). As candidates for further study we chose 11 SLSBs likely to have a mass ratio near unity and a velocity amplitude large enough to allow the primary and secondary spectra to be well separated.

In Figure 1 we illustrate some of the steps in our experimental reduction of a spectrum of a SLSB from the CfA echelle archive. In panel a we plot an echelle spectrum (well-exposed compared to the typical CfA spectrum) for the star G34-39, rectified to intensity (counted photons per $1.8 \mathrm{~km} \mathrm{~s}^{-1}$ pixel) versus wavelength (ngstroms); in panel b we plot the corresponding calculated spectrum chosen to match the temperature, gravity, metallicity, and rotational velocity of the primary; in panel $c$ we show the correlation of these two spectra; in panel $d$ we show the residual spectrum of the secondary left over after $80 \%$ of the counted photons in the observed spectrum (panel a), were subtracted away using the calculated spectrum (panel $b$ ) shifted to the velocity indicated by the initial correlation (panel c); finally, in panel e we show the correlation of the residual secondary spectrum (panel d) against the calculated spectrum (panel b). Note that the hint of a secondary peak which can be seen in the red wing of the primary peak in the initial correlation (panel $c$ ) is clearly revealed in the correlation of the residual secondary spectrum (panel e). 

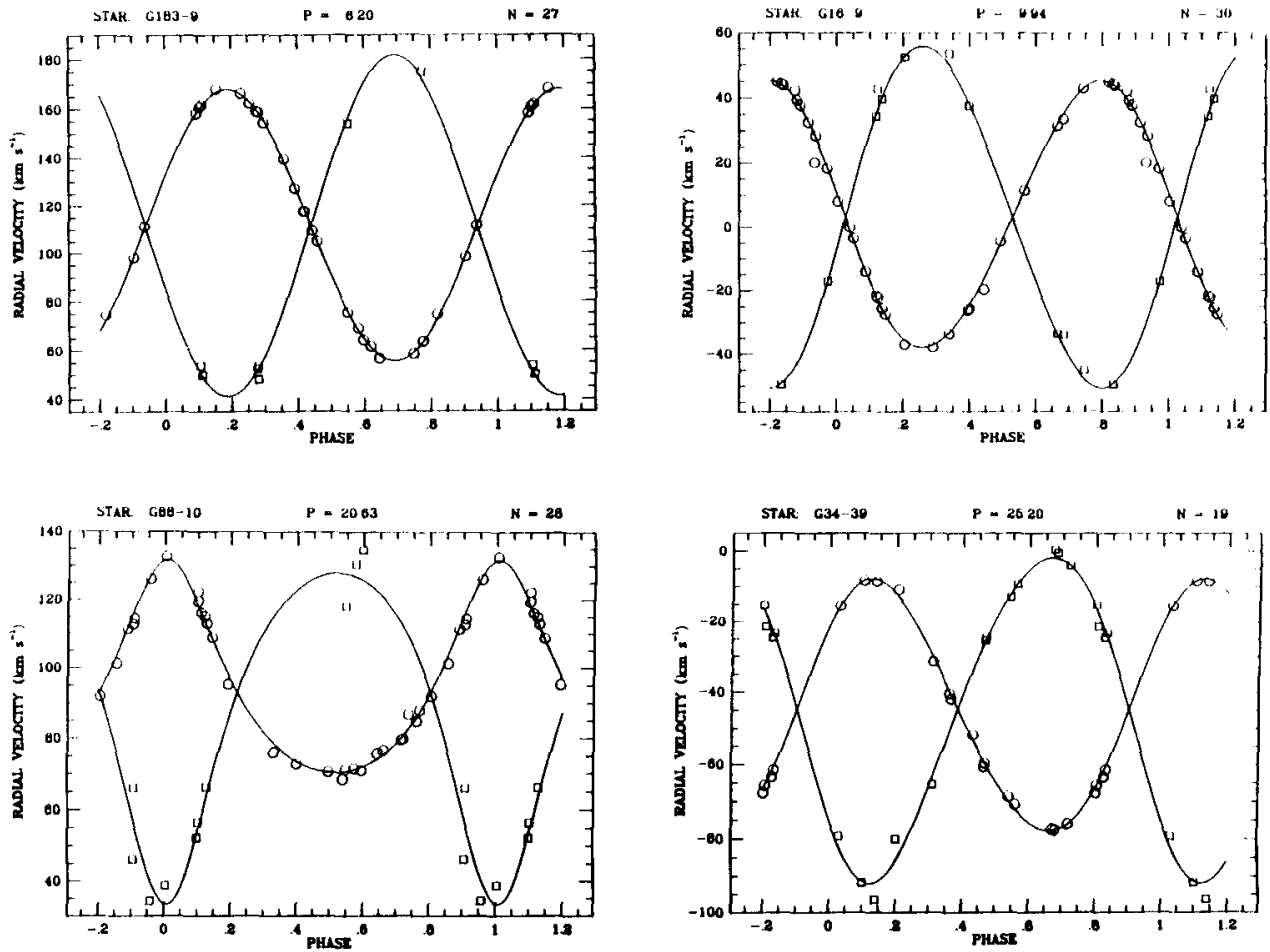

FIGURE 2. Velocity curves for 4 DLSBs with new orbital solutions.

For 6 of the 11 candidate SLSBs we were able to extract reliable velocities for the secondaries and to solve for DLSB orbits. Velocity curves for 4 of these are shown in Figure 2.

We thank Alejandra Milone, Jon Morse, and Guillermo Torres for their unselfish help with the synthetic spectra and correlations.

\section{REFERENCES}

Carney, B.W., Laird, J.B., Latham, D.W., \& Kurucz, R.L. 1987, AJ, 94, 1066

Duquennoy, A. \& Mayor, M. 1991, $A \mathcal{B} A, 248,485$

Latham D.W. 1992, this volume

Latham, D.W., Mazeh, T., Stefanik, R.P., Davis, R.J., Carney, B.W., Krymolowski, Y., Laird, J.B., Torres, G., \& Morse, J.A. 1992a, AJ, 104, 774

Latham, D.W., Mazeh, T., Stefanik, R.P., Davis, R.J., Carney, B.W., Torres, G., \& Laird, J.B. 1992b, this Colloquium

Mazeh, T. \& Goldberg, D. 1992a, ApJ, 394, 592

Mazeh, T. \& Goldberg, D. 1992b, this Colloquium

Torres, G. 1991, PhD thesis 